\title{
Surface-Modified Graphene Oxide with Compatible Interface Enhances Poly-L-Lactic Acid Bone Scaffold
}

\author{
Guoyong Wang, ${ }^{1}$ Chongxian He, ${ }^{1}$ Wengjing Yang, ${ }^{1}$ Fangwei Qi, ${ }^{1}$ Guowen Qian, \\ Shuping Peng $\mathbb{D}^{1,2}$ and Cijun Shuai $\mathbb{i D}^{1,3,4}$ \\ ${ }^{1}$ Institute of Bioadditive Manufacturing, Jiangxi University of Science and Technology, Nanchang 330013, China \\ ${ }^{2}$ School of Basic Medical Science, Central South University, Changsha 410083, China \\ ${ }^{3}$ State Key Laboratory of High Performance Complex Manufacturing, Central South University, Changsha 410083, China \\ ${ }^{4}$ Shenzhen Institute of Information Technology, Shenzhen 518172, China
}

Correspondence should be addressed to Cijun Shuai; shuai@csu.edu.cn

Received 7 February 2020; Accepted 21 February 2020; Published 7 March 2020

Academic Editor: Xiaoming Li

Copyright $\odot 2020$ Guoyong Wang et al. This is an open access article distributed under the Creative Commons Attribution License, which permits unrestricted use, distribution, and reproduction in any medium, provided the original work is properly cited.

Graphene oxide (GO) usually serves as a reinforce phase in polymer because of its superior mechanical strength and high specific surface area. In this work, GO was grafted with L-lactic acid monomer (denoted as GO@PLLA) to overcome the aggregation in matrix and then incorporated into the poly-L-lactic acid (PLLA) scaffold fabricated by selective laser sintering. In hybrid scaffold, GO@PLLA exhibited uniform dispersion in the matrix. Furthermore, mechanical interlock between GO@PLLA and PLLA matrix formed and reinforced the interface bonding. On the other hand, the heterogeneous distributed GO acted as effective nucleating agent and resultantly enhanced the crystallization. Results showed that the tensile and compressive strength of scaffolds increased by $143.3 \%$ and $127.6 \%$, respectively. Meanwhile, the scaffold exhibited an increased degradation rate of $37.9 \%$, which could be attributed to the abundant hydrophilic functional groups on GO. Moreover, the scaffold exhibited favorable bioactivity and biocompatibility. Herein, the developed hybrid scaffold showed potential capacity for bone tissue engineering.

\section{Introduction}

Poly-L-lactic acid (PLLA) has been receiving intensive attention in tissue engineering due to its especially favorable biocompatibility and degradability $[1,2]$. However, its insufficient mechanical properties handicap its profound application in bone tissue engineering [3-5]. Incorporating reinforcement phases to obtain a composite material is a feasible method to overcome this issue [6-8]. Nanofillers such as graphene oxide $[9,10]$, carbon nanotubes [11, $12]$, diamond nanoparticles $[13,14]$, and magnesium oxide nanoparticles [15-17] have been introduced into PLLA matrix to improve the mechanical performance. Amongst them, GO as a reinforcement phase was used to enhance the polymer due to its unique mechanical strength, including a strength of approximately $130 \mathrm{GPa}$ and modulus of approximately $1 \mathrm{TPa}$ [18]. In addition, the large specific surface area of GO is also conducive to its mechanical reinforcing effect [19]. What is more, GO exhibits suitable biological properties due to its abundant functional groups on the surface [20]. Nevertheless, the nanoreinforced effect of $\mathrm{GO}$ in polymer matrix was severely weakened because of its easy agglomeration in matrix [21,22].

Surface modification is an effective strategy to promote the dispersion of reinforcement phase in polymer [23-25]. Lactic acid monomer possesses carboxyl and hydroxyl double functional groups, which could bond with the active functional groups of GO. Thus, lactic acid monomer could be grafted on GO, which then polymerized into PLLA chain through liner condensation [26]. GO grafted with PLLA (denoted as GO@PLLA) was expected to mix well with PLLA matrix $[27,28]$. More significantly, GO@PLLA can intertwine with polymer matrix, which may form the mechanical interlock between matrix and GO@PLLA, thus effectively 
enhancing the interfacial bonding strength. For instance, Sun and $\mathrm{He}$ [29] reported that GO grafted with poly (D-lactide) (GO- $g$-PDLA) by the polymerization method. Subsequently, PLLA/GO- $g$-PLDA nanocomposites were prepared and enhanced the interfacial compatibility between GO-gPLDA and PLLA. Zhang et al. [30] proposed an approach to graft GO with poly (ethylene glycol) and then fabricated the composites nanofibrous scaffold via electrospinning technique, which exhibited enhanced the tensile strength and wettability.

Herein, GO@PLLA was prepared via in situ polycondensation method.GO@PLLA was then incorporated into PLLA to prepared hybrid scaffolds by selective laser sintering (SLS) [31]. Comparing with traditional methods, such as fused deposition modeling [32], electrospinning technique [33], and foaming technique [34], SLS exhibits great advantages including precise control of porous structure and personalized customization of bone scaffold. Then, the microstructure, mechanical properties, and degradation properties were evaluated. The enhancement mechanism was discussed and clarified. Furthermore, cell adhesion and proliferation were performed to verify the hybrid scaffolds as a potential candidate for bone regeneration.

\section{Materials and Methods}

2.1. Materials. GO was purchased from Chinese Academy of Sciences Chengdu organic chemistry Co. Ltd., China. PLLA powder $\left(2.0 \mathrm{dL} / \mathrm{g}, M_{\mathrm{w}}: 150 \mathrm{kDa}\right)$ was provided by Shandong Academy of Pharmaceutical Sciences Pilot Plant, China. The L-lactic acid aqueous solution $\left(90 \%, M_{\mathrm{w}}: 90.08 \mathrm{kDa}\right)$ was procured from Shanghai Aladdin Biochemical Technology Co. Ltd.

2.2. Preparation of GO@PLLA. GO@PLLA was prepared by in situ melt polycondensation in vacuum autoclave. The process to prepare GO@PLLA was demonstrated in Figure 1. A certain amount of GO $(0.5 \mathrm{~g})$ was added into the L-lactic acid aqueous solution $(100 \mathrm{~mL})$. The mixed solution was magnetically stirred 30 min under room temperature and then sonicated for $60 \mathrm{~min}$ to ensure GO homogeneously dispersed in the solution, followed by the adding of zinc oxide $(5 \mathrm{mg})$ into the solution as the initiator. The polycondensation process was executed in vacuum autoclave accompanied by magnetic stirring and the reaction at $120^{\circ} \mathrm{C}$ for $12 \mathrm{~h}$. After the polycondensation was completed, the condensation product was dissolved in excessive ethyl alcohol and centrifuged under the speed of $3000 \mathrm{r} / \mathrm{min}$. Abovementioned process was repeated until the unreacted substances were removed. The final production was dried at $55^{\circ} \mathrm{C}$ under vacuum until weight unchanged.

2.3. GO@PLLA Characterization. The variation of chemical structure of GO@PLLA was obtained by X-ray photoelectron spectrometer (XPS; Thermo ESCALAB 250XI, USA). The thermal stability was evaluated by a thermal gravimetric analyzer (TGA, 209 F3 Tarsus Netzsch). Each sample was heated from room temperature to $800^{\circ} \mathrm{C}$ at a rate of $10^{\circ} \mathrm{C} / \mathrm{min}$ in nitrogen environment. After keeping for $3 \mathrm{~min}$ to eliminate heat history, the samples were cooled to room temperature. The weight loss was recorded. Fourier-transform infrared (FTIR; Bruker Vertex 70 spectrometer) spectrometer was employed to measure the functional groups of composites within the range of 4000 to $400 \mathrm{~cm}^{-1}$. Raman spectra of the composites were acquired by a Raman microscope system (LabRam HR Evolution, Horiba) equipped with a laser wavelength of $532 \mathrm{~nm}$ and laser power of $20 \mathrm{~mW}$. The dispersions of GO and GO@PLLA in alcohol were observed via solubility test. $0.5 \mathrm{mg}$ GO and GO@PLLA were, respectively, added into $50 \mathrm{~mL}$ alcohol to prepare homogenous suspension with stirring and ultrasonic. The suspension state was observed immediately and static after 12 hours.

2.4. Preparation PLLA and Its Hybrid Scaffolds. PLLA, PLLA/GO (1.0 wt.\%), and PLLA/GO@PLLA (1.0wt.\%) composite powders were prepared through a series of processes involved dissolving, magnetic stirring, sonicating, centrifuging, and vacuum drying. A self-designed 3D printing system was used to prepare the scaffolds. The preparation procedure was as follows: the scaffold model was imported into 3D printing system after designing and then converted to STL files. Consequently, the laser beam scanned the printing area selectively layer by layer according to the scaffold model. The $3 \mathrm{D}$ printing was performed at a series of optimum parameters: laser power $3.5 \mathrm{~W}$, scanning speed $180 \mathrm{~mm} / \mathrm{s}$, scanning interval of $0.1 \mathrm{~mm}$, and powder-bed depth of $0.1 \mathrm{~mm}$. The scaffolds were obtained with approximately $600 \mu \mathrm{m}$ pore size and $450 \mu \mathrm{m}$ strut size.

2.5. Microstructural Characterization. The crystallization of PLLA, PLLA/GO, and PLLA/GO@PLLA scaffolds were analyzed on a differential scanning calorimetry (DSC; 214Polyma, Netzsch) accompany with a $50 \mathrm{~mL} / \mathrm{min}$ nitrogen pumping. The composites (about $10 \mathrm{mg}$ ) were heated from room temperature to $200^{\circ} \mathrm{C}$ at a heating rate of $10^{\circ} \mathrm{C} / \mathrm{min}$. $\mathrm{X}$-ray diffraction spectra of PLLA, PLLA/GO, and PLLA/GO@PLLA composites were acquired by an X-ray diffractometer (XRD; X'Pert PRO MPD). In detail, the specimen of the scaffolds was cut to thin slices $\left(10 \times 20 \times 15 \mathrm{~mm}^{3}\right)$ and was scanned at the diffraction ranging from 10 to $80^{\circ}$ at a scanning speed of $2 \% \mathrm{~min}$. Fourier-transform infrared (FTIR) spectra within a reflectance mode of $4000-400 \mathrm{~cm}^{-1}$ were obtained to determine the surface properties of composite scaffolds. Raman spectrometer was used to detect the functional group of scaffolds with a wavelength of $532 \mathrm{~nm}$ at the range from 400 and $2500 \mathrm{~cm}^{-1}$.

The brittle fracture surface morphologies of PLLA and its hybrid scaffolds were observed by scanning electron microscope (SEM; Nova 450). The specimens were coated with gold for $150 \mathrm{~s}$ using a high-vacuum sputter coater ion sputter before SEM observation.

2.6. Mechanical Properties Evaluation. The universal testing machine (WD-D1, LTD, China) was used to evaluate the mechanical properties of PLLA and its hybrid scaffolds. Tensile tests were performed at a speeding of $2 \mathrm{~mm} / \mathrm{min}$. All tensile specimens were manufactured to dumbbell shapes $\left(13 \times 5 \times 2 \mathrm{~mm}^{3}\right)$. Compression tests were detected at a speed 


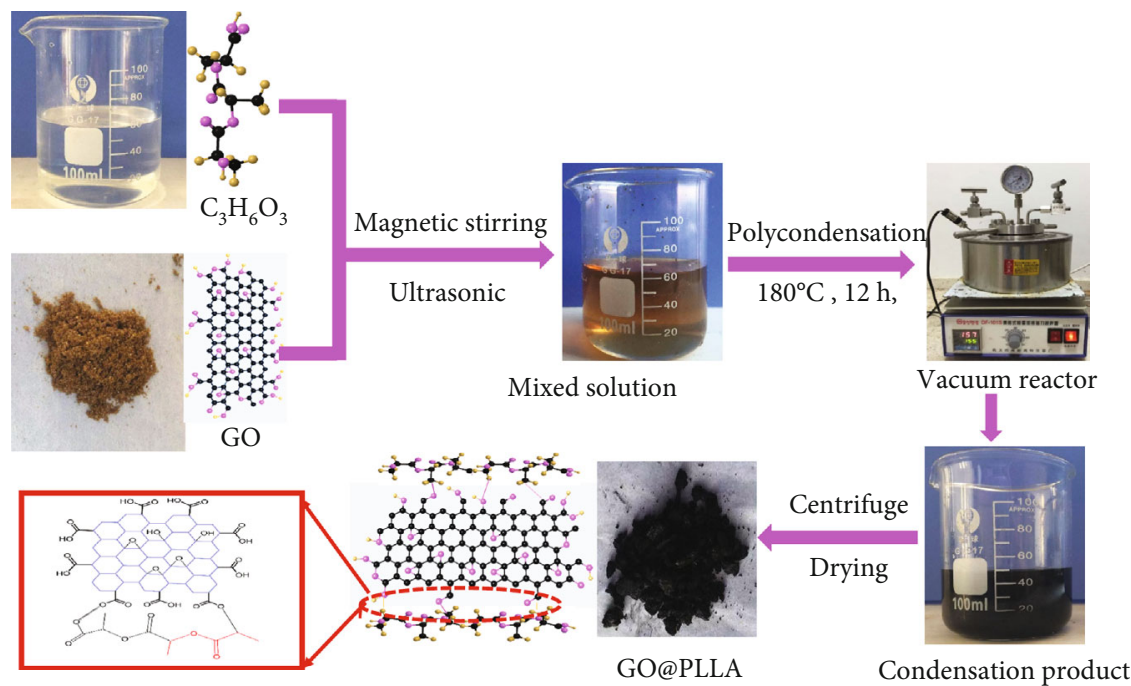

FIgURE 1: Flow diagram of synthesizing of GO@PLLA.

of $2 \mathrm{~mm} / \mathrm{min}$ according to GB/T1041-92 standard. Accordingly, all compression specimens were printed to square shapes with $5 \mathrm{~mm}$. Three times testing were performed during the measurement.

2.7. In Vitro Degradation Measurement. The degradation behavior of PLLA and its hybrid scaffold was evaluated in the phosphate-buffered saline (PBS) solution at room temperature. The initial specimens of PLLA and its hybrid scaffolds were weighed by a balancing scale as the control group. At 1, 2, 3, and 4 weeks, the specimens were taken out and washed with distilled water to erase the residual. After that, the specimens were dried in a vacuum oven at $55^{\circ} \mathrm{C}$ for $12 \mathrm{~h}$ and the residual weight of specimens was weighted. The residual weight fraction $(\Phi)$ was calculated by the following:

$$
\Phi=\frac{W \mathrm{i}}{W \mathrm{r}} \times 100 \%
$$

where $W_{\mathrm{i}}$ and $W_{\mathrm{r}}$ are the initial weight and the residual weight of specimens after incubation, respectively.

2.8. Cell Tests. MG-63 cells were used to perform the cell culture experiments. In detail, the cells are maintained in Dulbecco's modified Eagle's medium at $37^{\circ} \mathrm{C}$ in a $\mathrm{CO}_{2}$ condition. The culture medium was charged every 2 days. Prior to cell culture on the scaffolds, the scaffold samples $(10 \times 10 \times$ $2 \mathrm{~mm}^{3}$ ) were washed with PBS solution and sterilized with ultraviolet light for $0.5 \mathrm{~h}$ and then placed in 12 -well plates. After that, cell suspension at a density of $2 \times 10^{5}$ cells $/ \mathrm{mL}$ was seeded on the surface of the scaffolds. Meanwhile, enough culture medium was added to each well of the plate. The medium was changed each day during culture period. After culturing for 1, 4, and 7 days, the samples were taken out and rinsed thrice with PBS to remove residue and then gradient dehydrated with ethanol. The dehydrated samples were dried in a vacuum oven at $55^{\circ} \mathrm{C}$ for $24 \mathrm{~h}$. Finally, the cell-cultured samples were gold-coated for $2 \mathrm{~min}$ to observe cell morphology via SEM.

Live/dead cell staining assay was executed to evaluate cell spreading on the scaffold samples. After culturing for 1, 4, and 7 days, the samples were washed gently with PBS and immersed in $4 \%$ formaldehyde at $37^{\circ} \mathrm{C}$ for $20 \mathrm{~min}$. The fixed cells were stained with $2 \mu \mathrm{g} / \mathrm{mL}$ calcein acetoxymethyl ester and $1 \mu \mathrm{g} / \mathrm{mL}$ ethidium homodimer at $37^{\circ} \mathrm{C}$ for $20 \mathrm{~min}$, respectively. Then, the stained cells were dehydrated gradually with ethanol and dried under vacuum. Finally, the scaffolds fixed with stained cells were imaged by fluorescence microscope (Olympus, BX63).

Cell viability on PLLA and its hybrid scaffolds were evaluated through cell counting kit-8 assay (CCK-8). The MG-63 cell was cultured on the scaffolds for the desired time before the CCK- 8 experiment. After the culture medium was removed, CCK-8 reagent was added into each plate. Cells were further incubated at $37^{\circ} \mathrm{C}$ for another $4 \mathrm{~h}$. Finally, the optical density (OD) value at $450 \mathrm{~nm}$ was recorded by a spectrophotometric microplate reader.

2.9. Statistical Analysis. All the experiment was performed independently in triplicate to exhibit the difference between the different groups. The data was represented as means \pm standard deviation with the one-way ANOVA. Data with ${ }^{*} p<0.05$ present significant difference.

\section{Results and Discussion}

3.1. Characteristic of GO and GO@PLLA. XPS was employed to corroborate the changes of chemical functional groups for GO@PLLA. The peeks of functional groups of GO were exhibited in Figure 2(a). There were five peaks at $284.5 \mathrm{eV}$, $285.3 \mathrm{eV}, 287.0 \mathrm{eV}, 288.2 \mathrm{eV}$, and $289.3 \mathrm{eV}$, which were assigned to the functional groups of $\mathrm{C}=\mathrm{C} / \mathrm{C}-\mathrm{C}, \mathrm{C}-\mathrm{OH}, \mathrm{C}-\mathrm{O}$, $\mathrm{C}=\mathrm{O}$, and $\mathrm{O}-\mathrm{C}=\mathrm{O}$, respectively [24]. The spectrum of GO@PLLA was presented in Figure 2(b). There were six peaks. The component of $-\mathrm{OH}$ of $\mathrm{GO}$ was reduced 


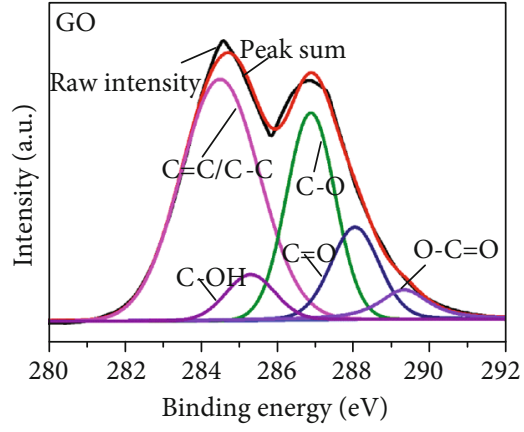

(a)

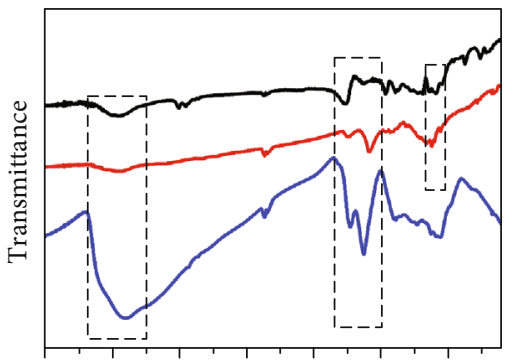

4000350030002500200015001000

Wavenumbers $\left(\mathrm{cm}^{-1}\right)$

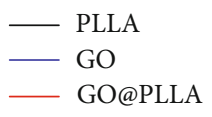

(d)
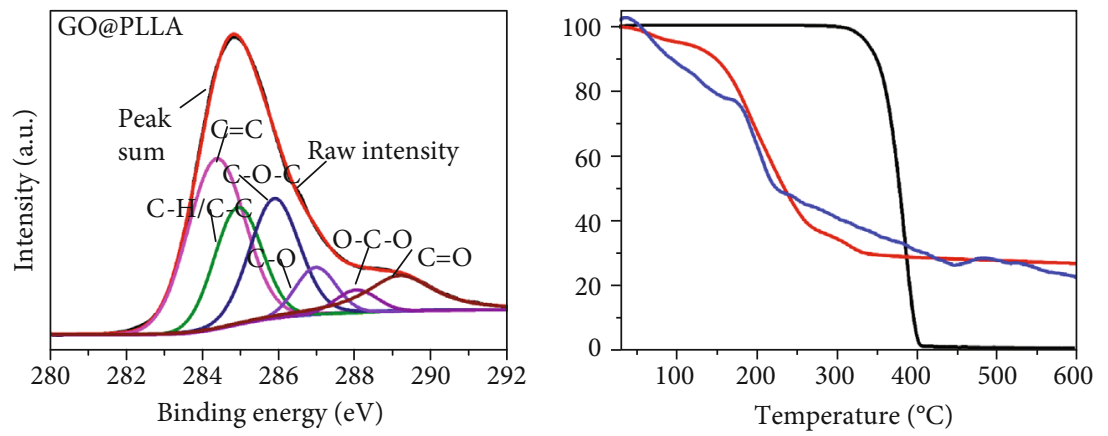

$$
\begin{aligned}
& - \text { PLLA } \\
& - \text { GO } \\
& - \text { GO@PLLA }
\end{aligned}
$$

(b)

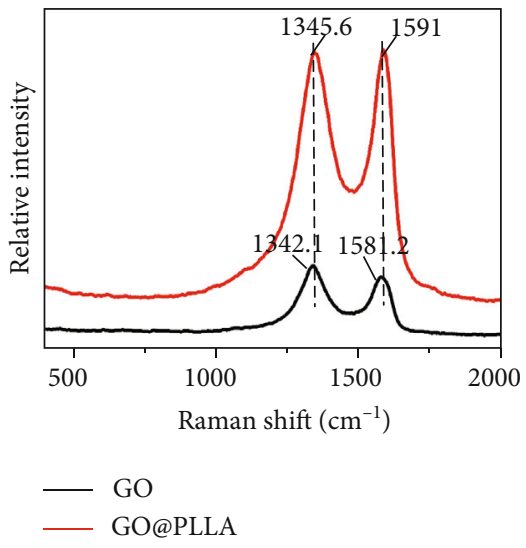

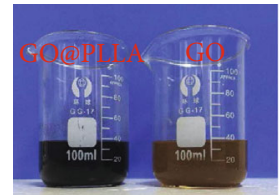

$0 \mathrm{~h}$ (c)

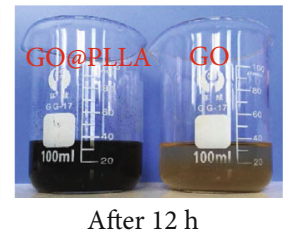

(f)

FIGURE 2: (a) XPS spectra of GO, (b) XPS spectra of GO@PLLA, (c) thermal degradation curves, (d) FTIR spectra, (e) Raman spectra of GO and GO@PLLA, and (f) photos of GO and GO@PLLA dispersed in ethanol after $12 \mathrm{~h}$.

significantly. Conversely, the components of $\mathrm{C}-\mathrm{H}$ and $\mathrm{O}=\mathrm{C}-$ O of GO@PLLA were increased due to the grafted PLLA chains. Furthermore, the intensity of $\mathrm{C}-\mathrm{OH}$ peak exhibited a relative decrease, while the intensity of $\mathrm{C}-\mathrm{H}$ and $\mathrm{O}-\mathrm{C}=\mathrm{O}$ enhanced in some degree.

The thermal stabilities of GO, PLLA, and GO@PLLA were tested by TGA, as presented in Figure 2(c). For GO, the weight loss at the range of 50 to $150^{\circ} \mathrm{C}$ was ascribed to the evaporation of moisture content [35]. At the following stage, the mass loss was mainly attributed to that the surface functional groups decomposed into $\mathrm{CO}, \mathrm{CO}_{2}$, and $\mathrm{H}_{2} \mathrm{O}$ [36]. As for GO@PLLA, the weight loss of GO@PLLA which occurred at 50 to $160^{\circ} \mathrm{C}$ was caused by the evaporation of water. Subsequently, the pyrolysis turned slow due to the successfully grafted PLLA chain on graphene. In addition, it could be seen that the PLLA chain grafted on GO was pyrolyzed completely before $400^{\circ} \mathrm{C}$, which is consistent with the obtained TGA curve.

Figure 2(d) exhibits the FTIR spectrum of PLLA, GO, and GO@PLLA. For the spectra of GO, the characteristic peaks located at 3430,1728 , and $1632 \mathrm{~cm}^{-1}$ corresponded to the $\mathrm{O}-\mathrm{H}, \mathrm{C}=\mathrm{O}$, and $\mathrm{C}-\mathrm{O}$ stretching vibrations, respectively [37]. As for GO@PLLA, the peak intensity around $3430 \mathrm{~cm}^{-}$ ${ }^{1}$ decreased significantly. It was believed that the esterifica- tion reaction between functional groups of L-lactic acid and GO resulted in the reduction of hydroxyl groups. At the same time, the peak position of $\mathrm{C}=\mathrm{O}$ stretching vibrations was gradually blue shifted from 1728 to $1750 \mathrm{~cm}^{-1}$, which could be ascribed to the increased ester groups caused by the graft of PLLA chains on GO. In addition, some typical resonances peaks of PLLA appeared at the spectrum of GO@PLLA. Therefore, it was demonstrated that PLLA chains in situ grafted on GO.

The conformational changes of GO@PLLA were assessed by Raman spectroscopy, as exhibited in Figure 2(e). For the Raman spectra of GO, the bands of D and G around 1342.1 and $1581.2 \mathrm{~cm}^{-1}$ corresponded to the vibration of $\mathrm{sp} 2$ and sp3 bonded carbon atoms, respectively [38]. For GO@PLLA, the peaks located at 1345.6 and $1591 \mathrm{~cm}^{-1}$ indicated a blue shift of 3.5 and $9.8 \mathrm{~cm}^{-1}$, respectively, which was closely related with the reaction of PLLA chain and GO [39].

The sedimentation experiment was executed to test the dispersity of GO and GO@PLLA in anhydrous ethanol, with obtained images demonstrated in Figure 2(f). GO and GO@PLLA powders formed uniform dispersion in anhydrous ethanol after magnetic stirring and ultrasonic. However, GO precipitated from the solution and a layer of aggregation appeared after $12 \mathrm{~h}$. As a comparison, GO@PLLA 
still presented uniform dispersion state in suspension. This indicated that a strong interaction between organic solvent and GO@PLLA improved the dispersion.

Based on above results, it was confirmed that the PLLA chains in situ grafted on GO successfully. GO provided abundant active sites for the grafted process due to its twodimensional nanosheet structure. Furthermore, GO served as initiator accelerated the reaction between macromolecule and surface-active groups. According to some literature, the monomer grafted on the GO surface induced by initiation or crystal during the initial step [36, 40]. Meanwhile, the formed free polycondensations coupled with the functional groups on GO. At last, GO was covered with PLLA chains with the continuous polycondensation reaction.

3.2. PLLA and Nanohybrid Scaffolds Properties. The SLS system and the as-prepared scaffold as shown in Figures 2(a) and 2(b). The uniform dispersion of the nanofiller and good interfacial compatibility between the nanofiller and the matrix play a positive role for the composites. In this study, dispersion of GO in matrix was observed via SEM, as shown in Figures 3(c)-3(f). The neat PLLA scaffold exhibited relatively smooth surface morphology, while PLLA hybrid scaffolds showed rougher surface morphologies. As showed in Figure 3(d), the aggregation of GO was observed and disjointed with the matrix, which might be attributed to the poor interfacial compatibility. For the PLLA/GO@PLLA scaffold, the well-distributed GO@PLLA firmly embedded in matrix, which exhibited enhanced interfacial bonding with PLLA matrix, as shown in Figure 3(e).

The FTIR spectrum of PLLA and hybrid scaffolds is presented Figure 4(a). The peak at $1753 \mathrm{~cm}^{-1}$ was shifted to high wavenumber, indicating slightly interaction due to the intermolecular and intramolecular hydrogen bonding between GO and the PLLA matrix. In addition, the shift of the peak was more significant for PLLA/GO@PLLA as compared to PLLA/GO. Raman spectroscopy was used to diagnose the chemical structure, as shown in Figure 4(b). The prominent peaks of the PLLA scaffold at 1769,1453 , and $873 \mathrm{~cm}^{-1}$ were observed, which assigned to $\mathrm{C}=\mathrm{O}$ stretching, $-\mathrm{CH} 3$ asymmetric deformation mode, and the $\mathrm{C}-\mathrm{COO}$ stretching, respectively. In PLLA hybrid scaffolds, the peak located at 1596 and $1345 \mathrm{~cm}^{-1}$ represented an intense tangential mode and a disorder induced mode of GO. For the PLLA/GO scaffold, the peaks of $\mathrm{C}=\mathrm{O}$ stretching and $-\mathrm{CH} 3$ asymmetric deformation mode shifted to 1772 and $1455 \mathrm{~cm}^{-1}$, respectively. It might be ascribed to that strong hydrogen interaction between PLLA and GO [41]. Furthermore, the upper shift was observed in the PLLA/GO@PLLA scaffold, which was caused by the stronger interaction between PLLA and GO@PLLA.

DSC was employed to evaluate the crystallization behavior of PLLA and its nanohybrids under nonisothermal condition. The second heating range from 30 to $200^{\circ} \mathrm{C}$ at the heating rate of $10^{\circ} \mathrm{C}$ was displayed in Figure 4(c). The melting temperature $\left(T_{\mathrm{m}}\right)$ of PLLA, PLLA/GO, and PLLA/GO@PLLA scaffolds was about 176.7, 178.5, and $180.1^{\circ} \mathrm{C}$, respectively. Obviously, after incorporating the nanofiller, the location of endothermic peak was slightly shifted. It might be attributed to mobility of the PLLA chains was restrained due to the effective combination between PLLA and GO [42]. Moreover, the thermal stability of PLLA/GO@PLLA was higher than PLLA/GO, which might be ascribed to uniform dispersion of GO@PLLA and interfacial bonding with PLLA matrix [43].

The influence of adding GO@PLLA on the crystalline structure of PLLA composites was analyzed by XRD, as presented in Figure 4(d). For the PLLA scaffold, two peaks typically located at $16.3^{\circ}$ and $18.6^{\circ}$ exhibited the crystallization in $\alpha$ form [44]. After incorporating the nanofiller into the PLLA scaffold, two peaks at 15.0 and $22.4^{\circ}$ were observed, which belong to $(001,210)$ reflection peaks of GO, respectively. Interestingly, it could be observed that the diffraction peaks of the PLLA hybrid scaffold blue shifted with incorporation of GO and GO@PLLA [45]. Meanwhile, the intensity of characteristic peak at $16.8^{\circ}$ increased for PLLA/GO@PLLA, which indicated an improved crystallinity due to stronger bonding between GO@PLLA and PLLA matrix.

3.3. Mechanical Properties. Bone scaffolds should possess suitable mechanical properties to withstand stress conduction in bone defect so as to provide support for the new bone growth [46, 47]. In this study, the tensile and compressive experiments were conducted to evaluate the mechanical strength of PLLA and its hybrid scaffolds. The stress-strain curves and tensile properties of the scaffolds are presented in Figure 5(a). For the PLLA/GO scaffolds, the tensile strength increased from 8 to $16 \mathrm{MPa}$, and the tensile modulus increased from 100 to $120 \mathrm{MPa}$, respectively. As for PLLA/GO@PLLA, the tensile strength and tensile modulus improved to $19 \mathrm{MPa}$ and $170 \mathrm{MPa}$, respectively. For the compression tests, the compressive strength of the PLLA/GO scaffold improved from 6 to $8 \mathrm{MPa}$ and the compression modulus increased to $80 \mathrm{MPa}$. More obviously, the compressive strength and modulus of the PLLA/GO@PLLA scaffold further improved to $16 \mathrm{MPa}$ and $190 \mathrm{MPa}$, respectively.

The mechanical properties of the PLLA/GO@PLLA scaffold are affected by many factors. Firstly, the grafted PLLA chains promoted homogenous dispersion of GO in the PLLA/GO@PLLA scaffold. Furthermore, GO@PLLA intertwined with PLLA matrix to form mechanical interlock, which promoted the better interfacial bonding. As a result, it suppressed the crack extension during tensile process through transferring the interior stress to GO sheets [48, 49], which could effectively bear the external force. In addition, the crystallinity of the PLLA scaffold has been improved after adding the GO@PLLA. Base on the above, significant improvement of mechanical properties was acquired. In the PLLA/GO scaffold, the GO tended to aggregate in PLLA matrix due to its higher specific surface energy, which might be the main reason for the undesirable enhancement.

3.4. In Vitro Degradation Behavior. The in vitro degradation property of PLLA and its hybrid scaffolds were evaluated. Figure 6(a) exhibits residual weight fraction of the scaffold during immersion test. The mass loss of PLLA reached 7.95\% after 4 -week immersion. Compared with the PLLA scaffold, the hybrid scaffolds exhibit obviously promoted 


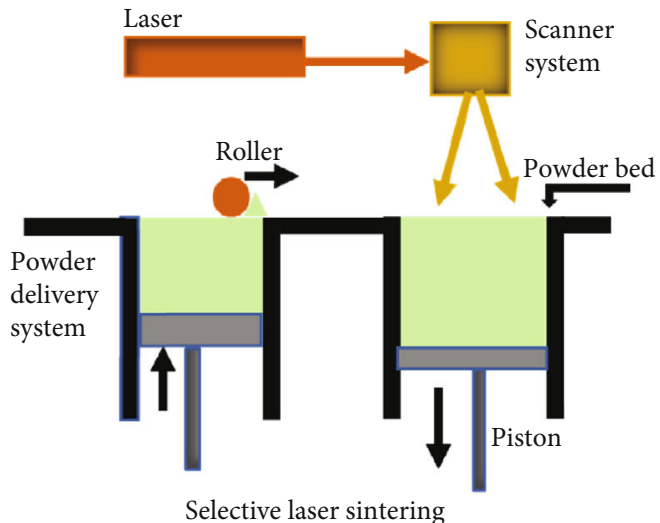

(a)

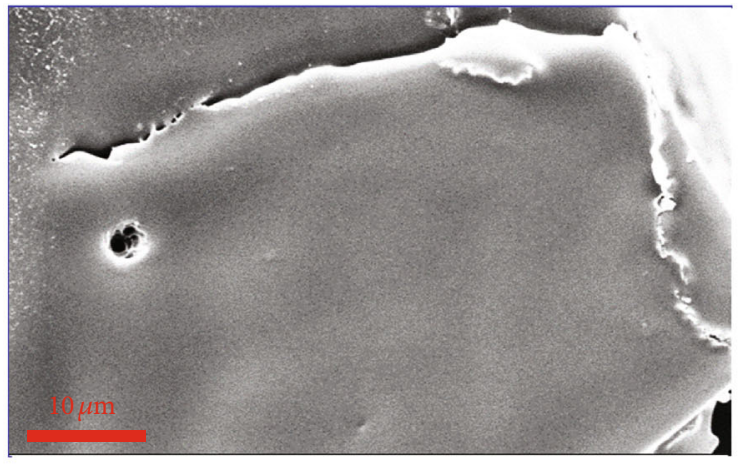

(c)

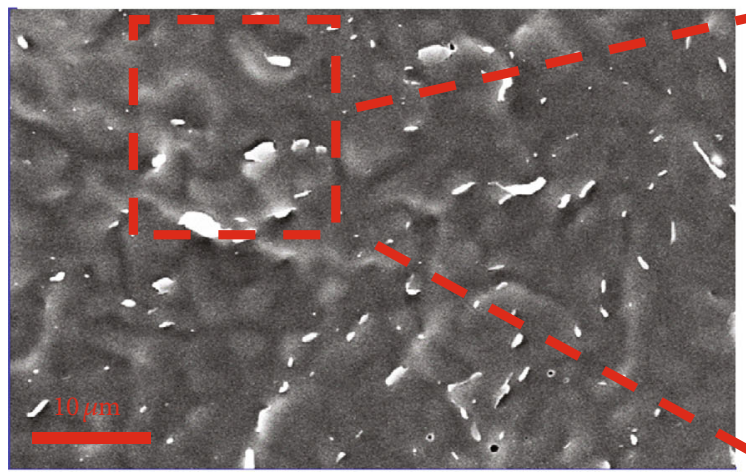

(e)
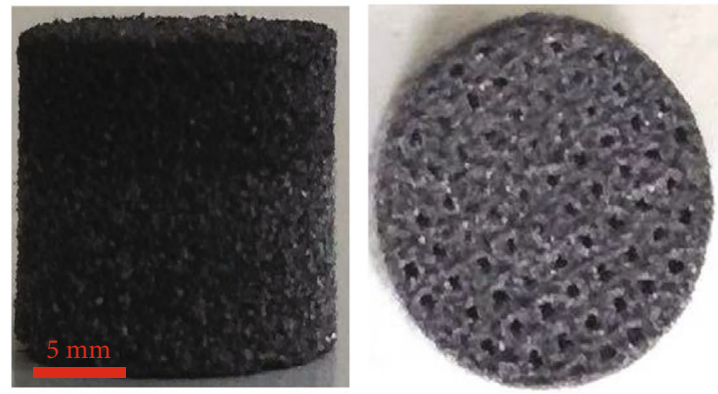

(b)

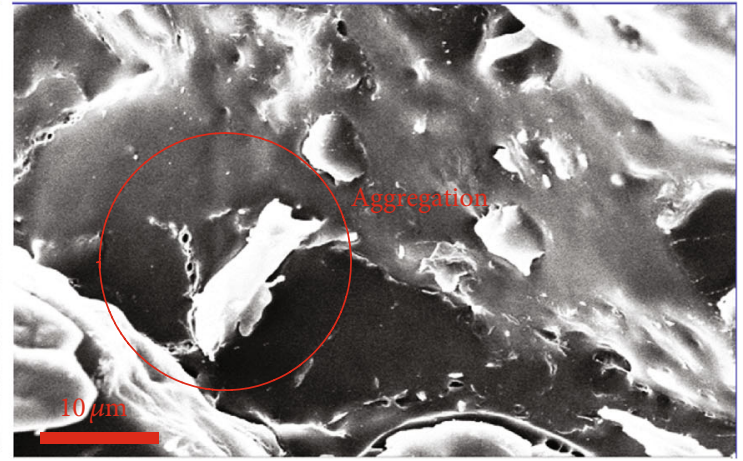

(d)

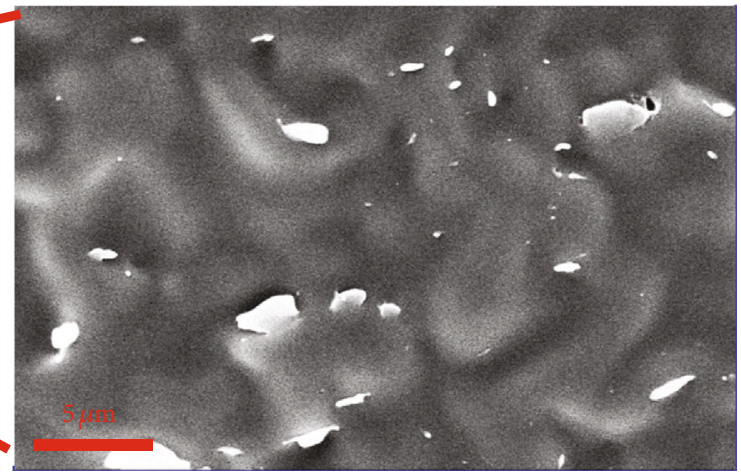

(f)

FIgURe 3: (a) The schematic diagram of SLS and (b) as-prepared scaffold. The brittle surface morphologies of (c) PLLA, (d) PLLA/GO, and (e) PLLA/GO@PLLA scaffolds. (f) Magnified image of (e).

degradation rate. The mass loss of PLLA/GO and PLLA/GO@PLLA scaffolds was $9.80 \%$ and $10.97 \%$, respectively. The result indicated that GO expedited the degradation of the PLLA scaffold. The $\mathrm{pH}$ values of the residual PBS solution soaked with scaffolds were detected, with results shown in Figure 6(b). Corresponding to the initial PBS solution, the $\mathrm{pH}$ value decreased from 7.4 to $7.2,7.12$, and 7.1, respectively, for PLLA, PLLA/GO, and PLLA/GO@PLLA scaffolds.

The surface morphologies of the immersed scaffolds were observed via SEM to further characterize the degradation behavior. Figures 6(c)-6(e) show the surface morphologies of PLLA and its hybrid scaffolds after immersion for 4 weeks.
The PLLA scaffold exhibited the relatively smooth surface with some tiny holes. The PLLA/GO scaffold shown relatively coarse surface. Some cracks concentrated in a relatively concentrated area, which may due to the aggregation of GO. Comparing with the PLLA/GO scaffold, the PLLA/GO@PLLA scaffold shown a relatively uniform corrosion morphology, and the erosion was much more serious. The in vitro degradation of scaffolds was accelerated after adding GO, which might be explained by the hydrophilicity of hybrid scaffolds [50]. The addition of GO improved the hydrophilicity of PLLA scaffold, thereby the surface wettability of scaffold becomes better. Furthermore, uniformly 


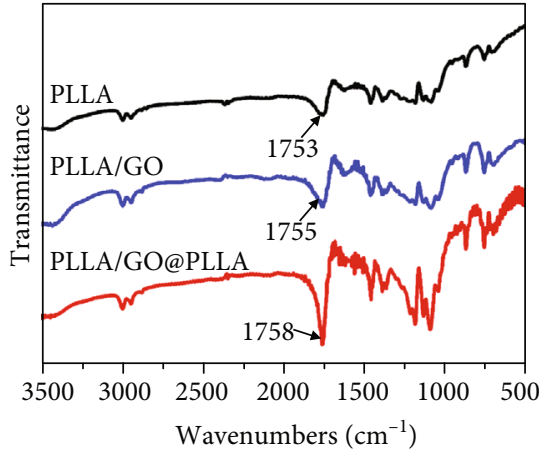

(a)

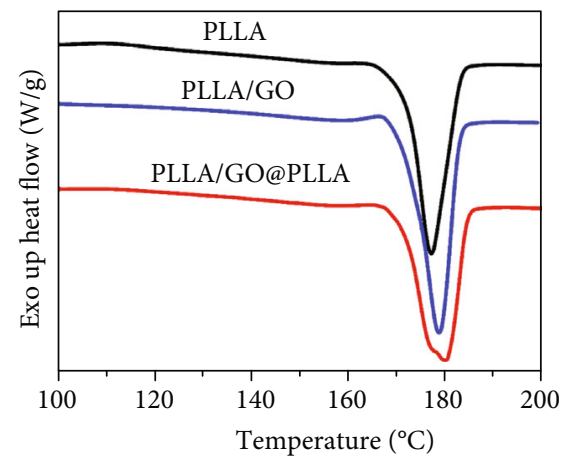

(c)

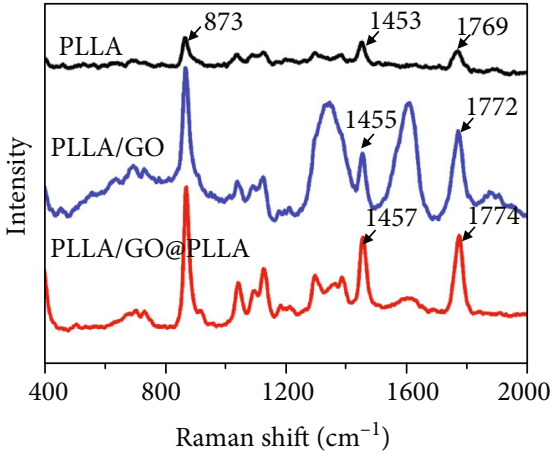

(b)

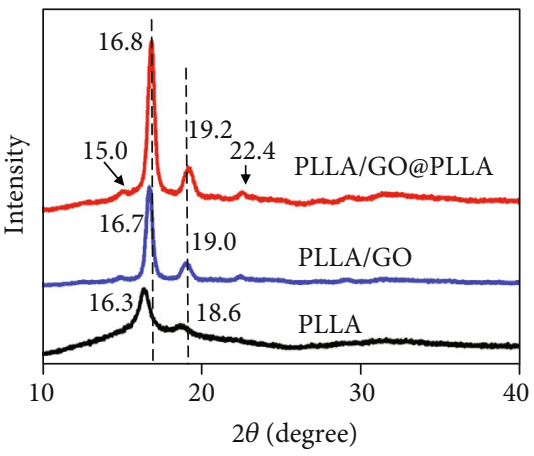

(d)

Figure 4: (a) FTIR, (b) Raman spectra, (c) DSC heating curves, and (d) XRD analysis of PLLA, PLLA/GO, and PLLA/GO@PLLA scaffolds.

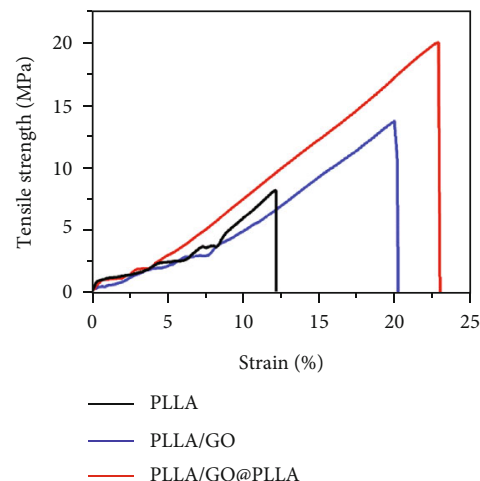

(a)

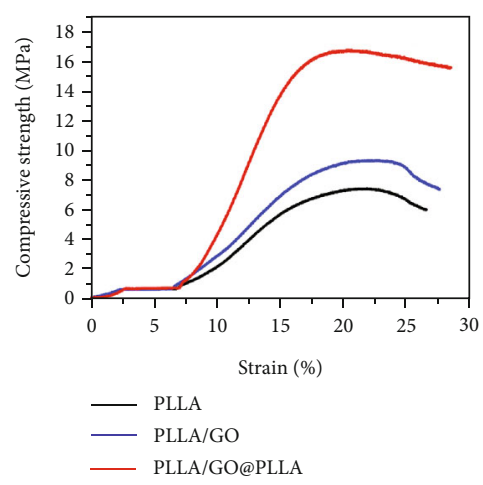

(d)

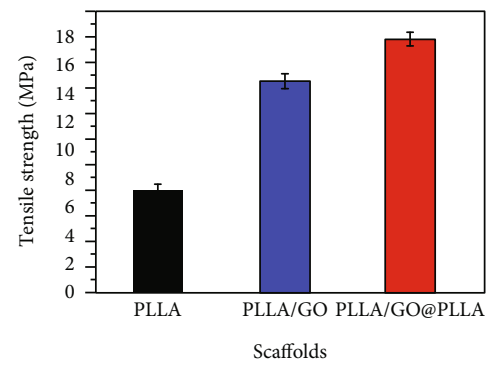

(b)

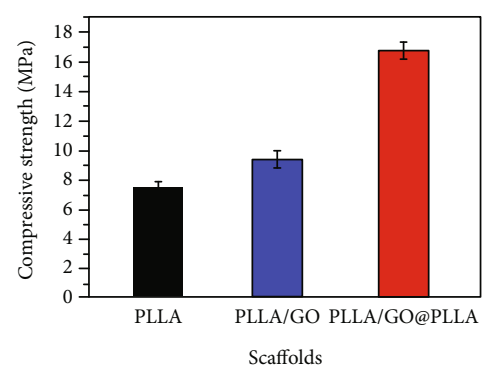

(e)

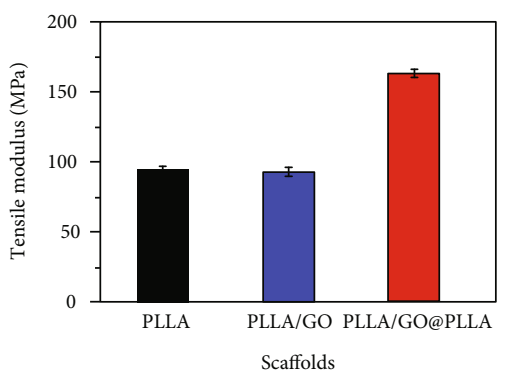

(c)

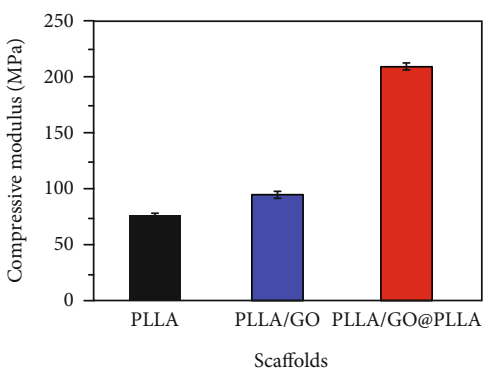

(f)

Figure 5: (a) Tensile stress-strain curves, (b) tensile strength, (c) tensile modulus, (d) compressive stress-strain curves, (e) compressive strength, and (f) compressive modulus of PLLA and its hybrid scaffolds. 


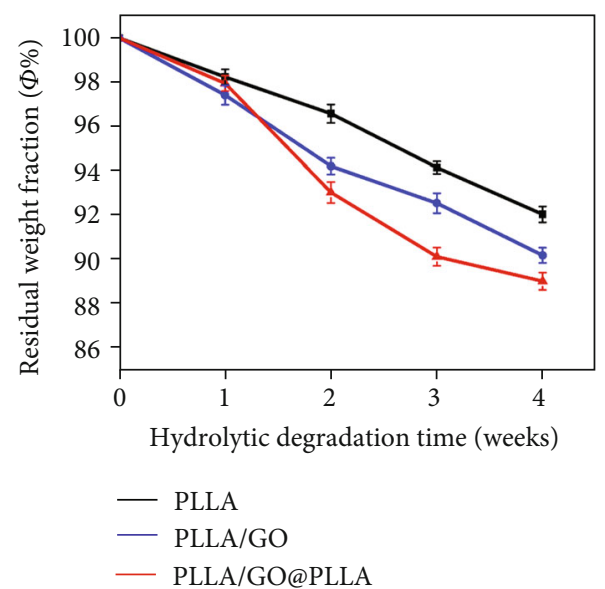

(a)

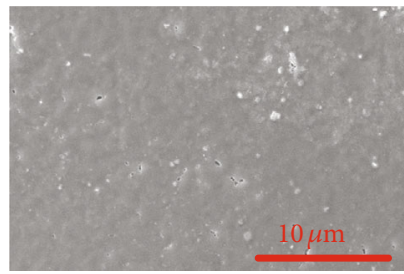

(c)

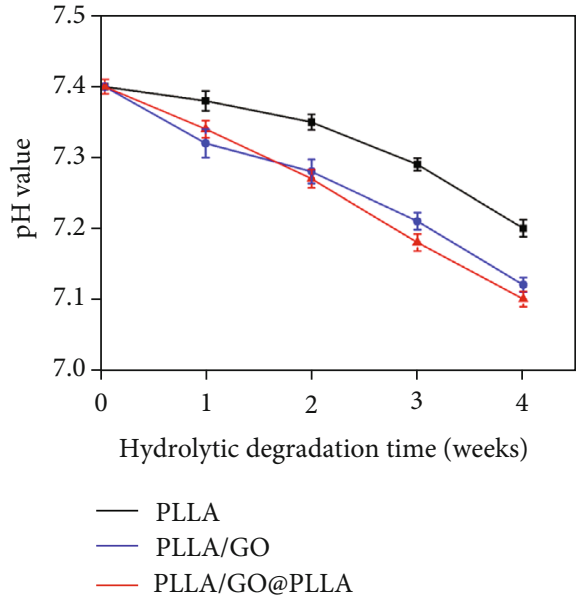

(b)

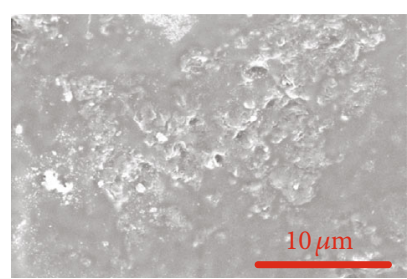

(d)

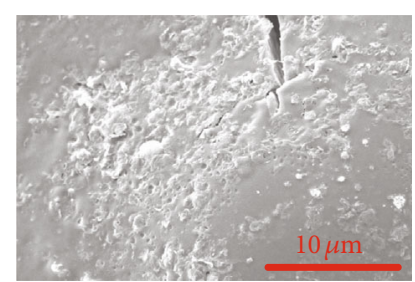

(e)

FIGURE 6: (a) Weight loss, (b) pH value, and SEM morphologies of (c) PLLA, (d) PLLA/GO, and (e) PLLA/GO@PLLA scaffolds after immersion in PBS solution.

dispersed GO and PLLA formed more two-phase interfaces in the scaffold, which provided more degrade sites and solution exchange channels for the PBS solution. It benefits the influx of the aqueous media into scaffolds [51,52].

3.5. Cytocompatibility. The SEM images of cells adhesion on different PLLA scaffolds after 1, 4, and 7 days were present in Figure 7. It could be seen that the cells fixed and spread on the scaffolds with culture time increasing. After culturing for 4 days, more cells and extracellular matrix exhibited typical morphologies and covered the surface of hybrid scaffolds. With the culturing time increased to 7 days, the cells on the PLLA/GO@PLLA scaffolds appeared mature and plumper shape [53]. It was indicated that incorporating GO@PLLA into scaffolds benefited cell adhesion and spreading.

Live and dead staining experiment was performed to further evaluate the biocompatibility of the PLLA and its hybrid scaffolds. The fluorescence images were presented in Figure 8. Almost no dead cells were observed in all scaffolds. Compared to PLLA and PLLA/GO scaffolds, more live cells were found in the PLLA/GO@PLLA scaffolds during the same period. It was indicated that the incorporation of GO@PLLA exhibited beneficial factor for cell growth. The CCK-8 assay was performed to quantitatively investigate the osteoblasts proliferation on the scaffolds, the results were present Figure 8(b). Obviously, the OD values increased gradually with culture period increasing for all scaffolds, and the addition of GO promoted cells proliferation. Notably, the OD values of the PLLA/GO@PLLA scaffold have significant difference in comparison to the PLLA scaffold

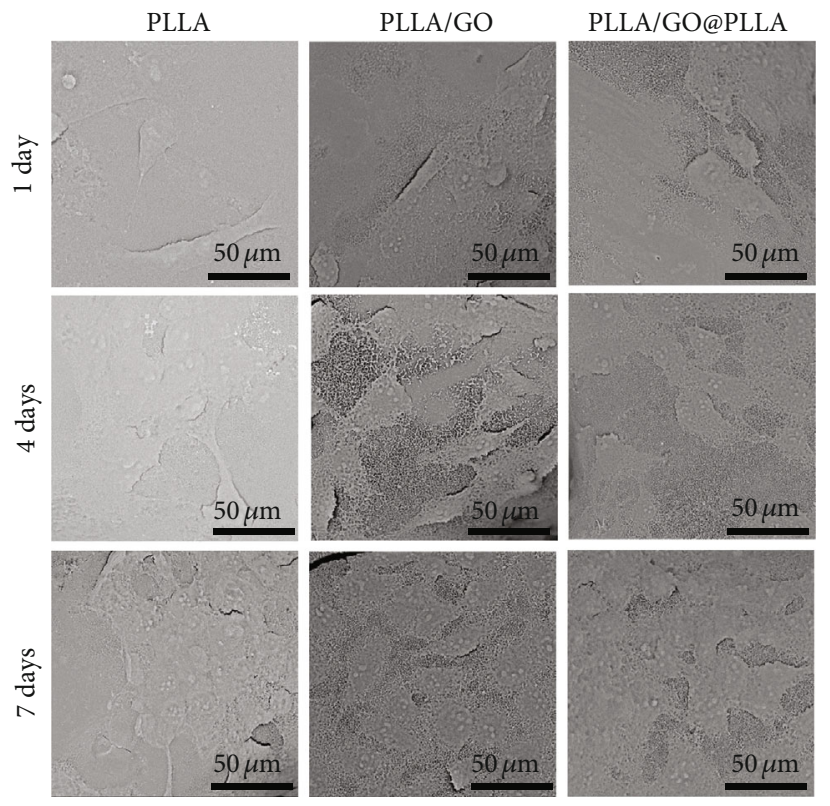

FIGURe 7: SEM micrographs of MG63 cells on PLLA and its hybrid scaffolds after 1, 4, and 7 days of culture.

throughout culture period, which showed higher cell viability. It was indicated that $\mathrm{GO}$ have intrinsic biocompatibility. Moreover, good dispersion in matrix after GO grafted with the PLLA chains, which may provide a suitable microenvironment for cells proliferation $[54,55]$. 


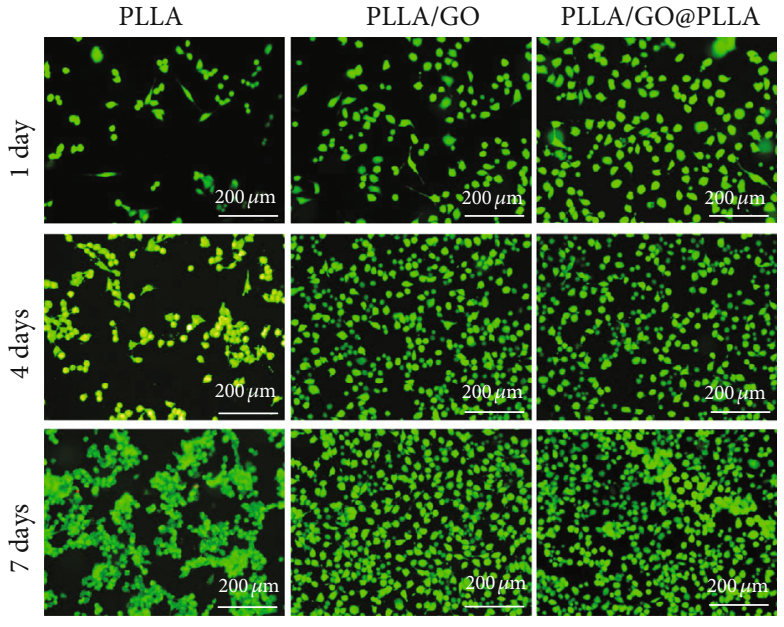

(a)

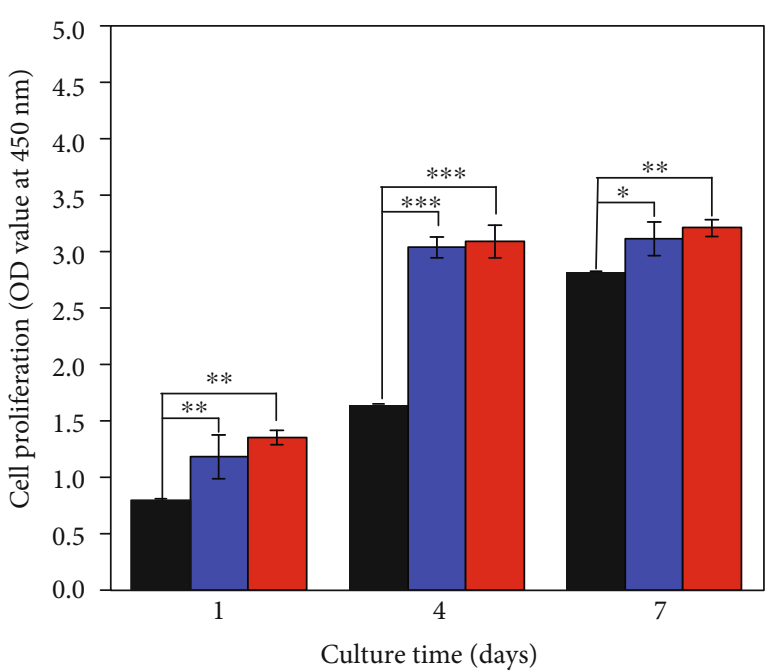

PLLA

PLLA/GO

PLLA/GO@PLLA

(b)

Figure 8: (a) Fluorescence images of MG-63 cells on PLLA and its hybrid scaffolds; and (b) OD determined by CCK-8 assays after culture for 1,4 and 7 days.

\section{Conclusions}

In this study, GO@PLLA was prepared through in situ polycondensation. PLLA and its hybrid scaffolds were successfully fabricated via SLS. GO@PLLA could uniformly dispersed in PLLA matrix. Moreover, stronger interface bonding was formed between GO@PLLA and PLLA matrix. As a result, the mechanical properties of the PLLA/GO@PLLA scaffold were significantly enhanced. Meanwhile, the weight loss tests indicated that GO enhanced the degradation rate of the PLLA scaffold. The cytocompatibility experiments showed that the PLLA/GO-p-PLLA scaffold exhibited favorable biological performance. Therefore, the PLLA/GO@PLLA scaffold was a potential substitute for bone tissue engineering.

\section{Data Availability}

The data used to support the findings of this study are available from the corresponding author upon request.

\section{Conflicts of Interest}

The authors declare that there are no conflicts of interest regarding the publication of this paper.

\section{Authors' Contributions}

Guoyong Wang and Chongxian He contributed equally to this work.

\section{Acknowledgments}

This study was supported by the following funds: (1) the Natural Science Foundation of China (51935014, 51905553, 81871494, 81871498, and 51705540); (2) Hunan Provincial Natural Science Foundation of China (2019JJ50774, 2018JJ3671, and 2019JJ50588); (3) Jiangxi Provincial Natural Science Foundation of China (20192ACB20005); (4) Guangdong Province Higher Vocational Colleges and Schools Pearl River Scholar Funded Scheme (2018); (5) the Open Sharing Fund for the Large-Scale Instruments and Equipments of Central South University; (6) the Project of Hunan Provincial Science and Technology Department (2017RS3008); and (7) the Project of Science and Technology of Jiangxi Provincial Education Department (GJJ190465).

\section{References}

[1] M. Eleuteri, L. Pastorino, and O. Monticelli, "On the degradation properties of electrospun fibers based on PLLA: the effect of a drug model modification," Polymer Degradation and Stability, vol. 153, pp. 109-117, 2018.

[2] K. Zhang, J.-K. Peng, Y.-D. Shi, Y.-F. Chen, J.-B. Zeng, and M. Wang, "Control of the crystalline morphology of poly (L-lactide) by addition of high-melting-point poly (L-lactide) and its effect on the distribution of multiwalled carbon nanotubes," The Journal of Physical Chemistry B, vol. 120, no. 30, pp. 7423-7437, 2016.

[3] N. Cai, Q. Dai, Z. Wang, X. Luo, Y. Xue, and F. Yu, “Toughening of electrospun poly (L-lactic acid) nanofiber scaffolds with unidirectionally aligned halloysite nanotubes," Journal of Materials Science, vol. 50, no. 3, pp. 1435-1445, 2015. 
[4] Q. Zhang, V. N. Mochalin, I. Neitzel et al., "Mechanical properties and biomineralization of multifunctional nanodiamondPLLA composites for bone tissue engineering," Biomaterials, vol. 33, no. 20, pp. 5067-5075, 2012.

[5] X. Li, J. Wei, K. E. Aifantis et al., "Current investigations into magnetic nanoparticles for biomedical applications," Journal of Biomedical Materials Research Part A, vol. 104, no. 5, pp. 1285-1296, 2016.

[6] C. Gao, M. Yao, C. Shuai, S. Peng, and Y. Deng, "Nano-SiC reinforced $\mathrm{Zn}$ biocomposites prepared via laser melting: Microstructure, mechanical properties and biodegradability," Journal of Materials Science \& Technology, vol. 35, no. 11, pp. 2608-2617, 2019.

[7] T. Qiao, P. Song, H. Guo, X. Song, B. Zhang, and X. Chen, "Reinforced electrospun PLLA fiber membrane via chemical crosslinking," European Polymer Journal, vol. 74, pp. 101108, 2016.

[8] M. Mariano, F. Pilate, F. B. de Oliveira et al., "Preparation of cellulose nanocrystal-reinforced poly (lactic acid) nanocomposites through noncovalent modification with PLLA-based surfactants," ACS Omega, vol. 2, no. 6, pp. 2678-2688, 2017.

[9] A. M. Pinto, J. Cabral, D. A. P. Tanaka, A. M. Mendes, and F. D. Magalhães, "Effect of incorporation of graphene oxide and graphene nanoplatelets on mechanical and gas permeability properties of poly (lactic acid) films," Polymer International, vol. 62, no. 1, pp. 33-40, 2013.

[10] P. Feng, J. Jia, S. Peng, W. Yang, S. Bin, and C. Shuai, "Graphene oxide-driven interfacial coupling in laser $3 \mathrm{D}$ printed PEEK/PVA scaffolds for bone regeneration," Virtual and Physical Prototyping, pp. 1-16, 2020.

[11] G. Wang, F. Qi, W. Yang et al., "Crystallinity and reinforcement in poly-L-lactic acid scaffold induced by carbon nanotubes," Advances in Polymer Technology, vol. 2019, article 8625325, 10 pages, 2019.

[12] M. Kaseem, K. Hamad, F. Deri, and Y. G. Ko, "A review on recent researches on polylactic acid/carbon nanotube composites," Polymer Bulletin, vol. 74, no. 7, pp. 2921-2937, 2017.

[13] P. Feng, Y. Kong, L. Yu et al., "Molybdenum disulfide nanosheets embedded with nanodiamond particles: co- dispersion nanostructures as reinforcements for polymer scaffolds," Applied Materials Today, vol. 17, pp. 216-226, 2019.

[14] R. Aversa, R. V. V. Petrescu, A. Apicella, and F. I. T. Petrescu, "Nano-diamond hybrid materials for structural biomedical application," American Journal of Biochemistry and Biotechnology, vol. 13, no. 1, pp. 34-41, 2017.

[15] D. J. Hickey, B. Ercan, L. Sun, and T. J. Webster, "Adding MgO nanoparticles to hydroxyapatite-PLLA nanocomposites for improved bone tissue engineering applications," Acta Biomaterialia, vol. 14, pp. 175-184, 2015.

[16] Y. Yang, J. Zan, W. Yang et al., "Metal organic frameworks as a compatible reinforcement in a biopolymer bone scaffold," Materials Chemistry Frontiers, vol. 4, no. 3, pp. 973-984, 2020.

[17] C. Shuai, J. Zan, Y. Yang et al., "Surface modification enhances interfacial bonding in PLLA/MgO bone scaffold," Materials Science and Engineering: C, vol. 108, p. 110486, 2020.

[18] W. Huang, "Graphene nanopapers," in Nanopapers, pp. 2758, Elsevier, 2018.

[19] X.-J. Shen, S. Yang, J.-X. Shen et al., "Improved mechanical and antibacterial properties of silver-graphene oxide hybrid/polylactid acid composites by in-situ polymerization," Industrial Crops and Products, vol. 130, pp. 571-579, 2019.
[20] A. L. Rivera-Briso, F. L. Aachmann, V. Moreno-Manzano, and Á. Serrano-Aroca, "Graphene oxide nanosheets versus carbon nanofibers: Enhancement of physical and biological properties of poly(3-hydroxybutyrate-co-3-hydroxyvalerate) films for biomedical applications," International Journal of Biological Macromolecules, vol. 143, pp. 1000-1008, 2020.

[21] W. Li, T. Shang, W. Yang et al., "Effectively exerting the reinforcement of dopamine reduced graphene oxide on epoxybased composites via strengthened interfacial bonding," ACS Applied Materials \& Interfaces, vol. 8, no. 20, pp. 1303713050, 2016.

[22] C. Wang, J. Li, S. Sun et al., "Electrophoretic deposition of graphene oxide on continuous carbon fibers for reinforcement of both tensile and interfacial strength," Composites Science and Technology, vol. 135, pp. 46-53, 2016.

[23] M. Xu, J. Zhu, F. Wang et al., "Improved in vitro and in vivo biocompatibility of graphene oxide through surface modification: poly (acrylic acid)-functionalization is superior to PEGylation," ACS Nano, vol. 10, no. 3, pp. 3267-3281, 2016.

[24] M. Yuan, Y. Chen, M. Yuan, H. Li, X. Xia, and C. Xiong, "Functionalization of graphene oxide with low molecular weight poly (lactic acid)," Polymers, vol. 10, no. 2, p. 177, 2018.

[25] C. Shuai, L. Yu, W. Yang, S. Peng, Y. Zhong, and P. Feng, "Phosphonic acid coupling agent modification of HAP nanoparticles: interfacial effects in PLLA/HAP bone scaffold," Polymers, vol. 12, no. 1, p. 199, 2020.

[26] L.-N. Wang, P.-Y. Guo Wang, and J.-C. Wei, "Graphene oxide-graft-poly (l-lactide)/poly (l-lactide) nanocomposites: mechanical and thermal properties," Polymers, vol. 9, no. 12, p. 429, 2017.

[27] W. Li, Z. Xu, L. Chen et al., "A facile method to produce graphene oxide- $g$-poly(L-lactic acid) as an promising reinforcement for PLLA nanocomposites," Chemical Engineering Journal, vol. 237, pp. 291-299, 2014.

[28] Y. Kang, C. Wang, X. Shi, G. Zhang, P. Chen, and J. Wang, "Crystallization, rheology behavior, and antibacterial application of graphene oxide- graft -poly (l-lactide)/poly (l-lactide) nanocomposites," Applied Surface Science, vol. 451, pp. 315324,2018

[29] Y. Sun and C. He, "Synthesis and stereocomplex crystallization of poly (lactide)-graphene oxide nanocomposites," ACS Macro Letters, vol. 1, no. 6, pp. 709-713, 2012.

[30] C. Zhang, L. Wang, T. Zhai, X. Wang, Y. Dan, and L.-S. Turng, "The surface grafting of graphene oxide with poly (ethylene glycol) as a reinforcement for poly (lactic acid) nanocomposite scaffolds for potential tissue engineering applications," Journal of the Mechanical Behavior of Biomedical Materials, vol. 53, pp. 403-413, 2016.

[31] C. Shuai, W. Yang, Y. Yang et al., "Selective laser melted FeMn bone scaffold: microstructure, corrosion behavior and cell response," Materials Research Express, vol. 7, no. 1, article 015404, 2020.

[32] M. C. Wurm, T. Möst, B. Bergauer et al., "In-vitro evaluation of Polylactic acid (PLA) manufactured by fused deposition modeling," Journal of Biological Engineering, vol. 11, no. 1, p. 29, 2017.

[33] F. Lopresti, F. Carfi Pavia, I. Vitrano, M. Kersaudy-Kerhoas, V. Brucato, and V. la Carrubba, "Effect of hydroxyapatite concentration and size on morpho-mechanical properties of PLA-based randomly oriented and aligned electrospun nanofibrous mats," Journal of the Mechanical Behavior of Biomedical Materials, vol. 101, article 103449, 2020. 
[34] J. Ju, X. Peng, K. Huang et al., "High-performance porous PLLA-based scaffolds for bone tissue engineering: Preparation, characterization, and in vitro and in vivo evaluation," Polymer, vol. 180, article 121707, 2019.

[35] S. Cheng, X. Chen, Y. G. Hsuan, and C. Y. Li, "Reduced graphene oxide-induced polyethylene crystallization in solution and nanocomposites," Macromolecules, vol. 45, no. 2, pp. 993-1000, 2012.

[36] J.-H. Yang, S.-H. Lin, and Y.-D. Lee, "Preparation and characterization of poly (L-lactide)-graphene composites using the in situ ring-opening polymerization of PLLA with graphene as the initiator," Journal of Materials Chemistry, vol. 22, no. 21, pp. 10805-10815, 2012.

[37] G. Z. Papageorgiou, Z. Terzopoulou, D. Bikiaris et al., "Evaluation of the formed interface in biodegradable poly(l-lactic acid)/graphene oxide nanocomposites and the effect of nanofillers on mechanical and thermal properties," Thermochimica Acta, vol. 597, pp. 48-57, 2014.

[38] S. S. Nanda, D. K. Yi, and K. Kim, "Study of antibacterial mechanism of graphene oxide using Raman spectroscopy," Scientific Reports, vol. 6, no. 1, article 28443, 2016.

[39] W. Huang, S. Wang, C. Guo, X. Yang, Y. Li, and Y. Tu, "Synthesis and characterization of well-defined poly(l-lactide) functionalized graphene oxide sheets with high grafting ratio prepared through click chemistry and supramolecular interactions," Polymer, vol. 55, no. 18, pp. 4619-4626, 2014.

[40] Y. Cao, T. Shi, C. Jiao et al., "Fabrication and properties of zirconia/hydroxyapatite composite scaffold based on digital light processing," Ceramics International, vol. 46, no. 2, pp. 2300-2308, 2020.

[41] X. Meng, V. Bocharova, H. Tekinalp et al., "Toughening of nanocelluose/PLA composites via bio-epoxy interaction: mechanistic study," Materials \& Design, vol. 139, pp. 188197, 2018.

[42] J. Li, P. Xiao, H. Li et al., "Crystalline structures and crystallization behaviors of poly(l-lactide) in poly(l-lactide)/graphene nanosheet composites," Polymer Chemistry, vol. 6, no. 21, pp. 3988-4002, 2015.

[43] Q. Li, S. Qin, X. Tian et al., "Enhancement of the interface in poly(l-lactide) and poly(propylidene carbonate) blends by introducing of poly(l-lactide)-grafted graphene oxide to improve mechanical properties," Applied Surface Science, vol. 433, pp. 739-749, 2018.

[44] M. C. Righetti, M. Gazzano, M. L. Di Lorenzo, and R. Androsch, "Enthalpy of melting of $\alpha$ ' - and $\alpha$-crystals of poly(l-lactic acid)," European Polymer Journal, vol. 70, pp. 215-220, 2015.

[45] J. Shi, W. Wang, Z. Feng, D. Zhang, Z. Zhou, and Q. Li, "Multiple influences of hydrogen bonding interactions on PLLA crystallization behaviors in PLLA/TSOS hybrid blending systems," Polymer, vol. 175, pp. 152-160, 2019.

[46] C. Shuai, Z. Zeng, Y. Yang et al., "Graphene oxide assists polyvinylidene fluoride scaffold to reconstruct electrical microenvironment of bone tissue," Materials \& Design, vol. 190, article 108564, 2020.

[47] Y. Yang, C. He, Dianyu E et al., "Mg bone implant: features, developments and perspectives," Materials \& Design, vol. 185, article 108259, 2020.

[48] D. Qian, E. C. Dickey, R. Andrews, and T. Rantell, "Load transfer and deformation mechanisms in carbon nanotube-polystyrene composites," Applied Physics Letters, vol. 76, no. 20, pp. 2868$2870,2000$.

[49] M. M. Gudarzi and F. Sharif, "Enhancement of dispersion and bonding of graphene-polymer through wet transfer of functionalized graphene oxide," Express Polymer Letters, vol. 6, no. 12, pp. 1017-1031, 2012.

[50] X.-c. Du, X.-1. Xu, X.-h. Liu, J.-h. Yang, Y. Wang, and X.-1. Gao, "Graphene oxide induced crystallization and hydrolytic degradation of poly(butylene succinate)," Polymer Degradation and Stability, vol. 123, pp. 94-104, 2016.

[51] J. Duan, Y.-n. Xie, J.-h. Yang et al., "Graphene oxide induced hydrolytic degradation behavior changes of poly(l-lactide) in different mediums," Polymer Testing, vol. 56, pp. 220-228, 2016.

[52] C. Shuai, Y. Cheng, Y. Yang, S. Peng, W. Yang, and F. Qi, "Laser additive manufacturing of $\mathrm{Zn}-2 \mathrm{Al}$ part for bone repair: formability, microstructure and properties," Journal of Alloys and Compounds, vol. 798, pp. 606-615, 2019.

[53] C. Gao, M. Yao, S. Li, P. Feng, S. Peng, and C. Shuai, "Highly biodegradable and bioactive Fe-Pd-bredigite biocomposites prepared by selective laser melting," Journal of Advanced Research, vol. 20, pp. 91-104, 2019.

[54] H. Liang, Y. Yang, D. Xie et al., "Trabecular-like Ti-6Al-4V scaffolds for orthopedic: fabrication by selective laser melting and in vitro biocompatibility," Journal of Materials Science \& Technology, vol. 35, no. 7, pp. 1284-1297, 2019.

[55] S. He, S. Yang, Y. Zhang et al., "LncRNA ODIR1 inhibits osteogenic differentiation of hUC-MSCs through the FBXO25/H2BK120ub/H3K4me3/OSX axis," Cell Death \& Disease, vol. 10, no. 12, p. 947, 2019. 